\title{
Effect of UV Radiation by Projectors on 3D Printing
}

\author{
laroslav Kovalenko ${ }^{1, a}$ and Maryna Garan ${ }^{1}$ \\ ${ }^{1}$ Department of Manufacturing Systems and Automation, Technical University of Liberec, 46117 Liberec, Studentska \\ 1402/2, Czech Republic
}

\begin{abstract}
Polymers that solidify under light radiation are commonly used in digital light processing (DLP) 3D printing. A wide range of photopolymers use photoinitiators that react to radiation in range of ultraviolet (UV) wavelength. In the present study we provided measurement of radiant fluence in the UV wavelength range from $280 \mathrm{~nm}$ to $400 \mathrm{~nm}$ for two data projectors and compared effect of radiation on quality of 3D printing. One projector is commonly used DLP projector with high energy lamp. Second one is an industrial projector, in which RGB light emitting diodes (LEDs) are replaced by UV LEDs with wattage at the level of $3.6 \%$ of the first one. Achieved data confirmed uneven distribution of radiant energy on illuminated area. These results validate, that undesired heating light causes internal stress inside built models that causes defects in final products.
\end{abstract}

\section{Introduction}

Additive manufacturing (AM) technology becomes more spread not only in prototyping but also in a small quantity production. For example, NASA uses it in space science for customizing tools, development of multifunction devices for life support and radiation shielding [1]. It's possible, because AM offers alternative approaches than traditional manufacturing.

This method of production starts its history in 1986 from process called Stereolithography (SL) [2]. It used an UV laser for solidifying of photopolymer resins. Laser is placed above the bath with build material. Laser beam moves on the surface and solidifies resin point by point. After finishing the layer, platform with solid material moves down and laser starts its next path. The main disadvantage of this method is necessity to have a full tank of photopolymer resin which is not cost-effective. Sony/DMEC company had started producing of commercial versions of SL printers in 1988 but stopped it just in 2007 [3]. Development of this technology raised mass production of photopolymer resins for SL. But progress wasn't still so fast. Machines were expensive and just few hundreds of them were sold.

Digital Light Processing (DLP) 3D printers work similarly to SL machines and use photosensitive polymers as build material. But instead of laser they use dynamic mask, which is generated by digital mirror device (DMD) [4]. Light beam from UV radiation generator goes through DMD chip, which can control each pixel in its matrix. It means, that we have "white" (illuminated) and "black" (nonilluminated) points on the surface. At "white" points polymerization starts, at "black" points polymer remains liquid. This way, the full layer can be solidified at the same time unlike laser stereolithography. Also using of bottom-up printing technology, which is shown on Figure 1, can

\footnotetext{
${ }^{\text {a }}$ Corresponding author : iaroslav.kovalenko@tul.cz
} 
reduce volume of needed material. One of the main advantages of DLP printers is high precision and building speed. In case of laser SL printer, one point is printed in one moment of time unlike DLP printer, when a whole layer is printed simultaneously. On the other hand, duration of radiation for one layer of DLP printer is longer than for one point of laser printer. Precision depends on resolution of DMD chip and size of build area. For example, with resolution of 1900 x 1200 pixels we can reach a precision of $20 \mu \mathrm{m}$ with size of build platform $38 \times 24 \mathrm{~mm}$ in XY direction.
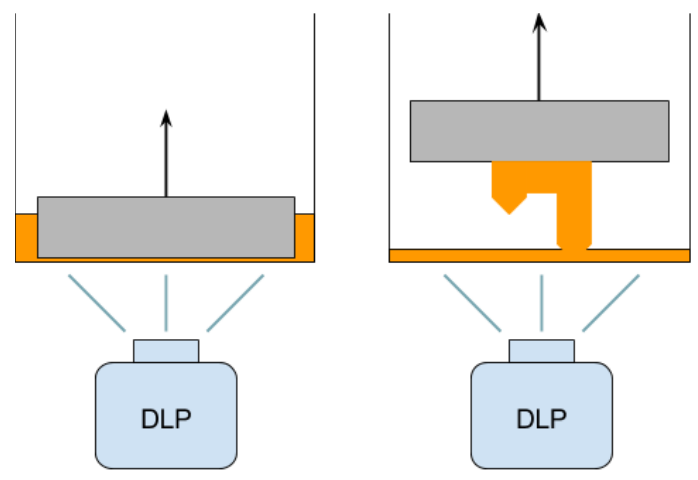

Figure 1. Schematic sketch of bottom-up 3D DLP printers

\section{Photopolymer resins}

Using of photopolymer solutions makes possible to add additional components, that can change properties of the solution, and consequently of built model. For obtaining of micro-structured surfaces with high precision, additional ceramic or metallic powders may be used. These additives serve for reaching of better mechanical properties of the model [5]. A lot of different methods in this area were designed and experimentally approved by TU Wien. Experiments including ceramic powders to photopolymer solution were made using alumina [6-9], silica [6], Bioglass ${ }^{\circledR}[7,8]$ and zirconia $[7,9]$. Different amounts of additives were added to the photopolymer solutions, mixed and used like a build material for a different types of SL printers. These solutions were used for printing of green bodies, which were sintered after.

Photopolymer solutions consist of three basic elements: monomers/oligomers, photo initiators and blockers [10]. Using of different components and different proportions of basic components makes a big variety of existing resins. Monomers/oligomers are basic components, which mostly have above $95 \mathrm{vol} \%$ of the solution. Photo initiators determine range of radiation, which can be used for solidifying, and curing speed. The higher percentage of initiator, the faster reaction is. Blockers define a deepness of radiation penetration and consequently have effect on layer thickness. For start of polymerization, photo initiators are exposed by light. Different initiators are sensible to specific wavelengths [11]. Typically for DLP 3D printers, resins that are sensible to UV wavelength are used. Common hand-made DLP printers for solidifying of photopolymer solution use office projectors. They are cost-effective, but mostly they have UV wavelength as unwanted output. In our research we compare radiant energy, radiated the experimental surface, from common office DLP projector and industrial projector with UV LEDs. 


\section{Experimental setup}

For this test we used widespread office projector Acer H6510BD and well-known industrial projector DLP LightCrafter E4500 (Texas Instruments Inc.) with replaced lamp for UV LED (EKB Technologies Ltd.). Main properties of compared projectors are presented in Table 1.

Table 1. Main properties of tested projectors

\begin{tabular}{|l|l|l|}
\hline Name & Acer H6510BD & LightCrafter E4500 \\
\hline Native Resolution & $1920 \times 1080(\mathrm{FHD})$ & $1280 \times 800(\mathrm{WXGA})$ \\
\hline Aspect Ratio & $16: 9$ & $16: 10$ \\
\hline Size (H X X D) & $80 \mathrm{~mm} \times 220 \mathrm{~mm} \times 260 \mathrm{~mm}$ & $48 \mathrm{~mm} \times 140 \mathrm{~mm} \times 152 \mathrm{~mm}$ \\
\hline Lamp Wattage & $210 \mathrm{~W}$ & $7.5 \mathrm{~W}$ \\
\hline Lamp Wavelength & Visible spectrum & $385-405 \mathrm{~nm}$ \\
\hline Throw Ratio & $1.15: 1-1.50: 1$ & $1.2: 1$ \\
\hline Offset & $29 \%$ & $0 \%$ \\
\hline Min. Image Size & $53.3 \mathrm{~mm} \times 30 \mathrm{~mm}$ & $20.4 \mathrm{~mm} \times 12.7 \mathrm{~mm}$ \\
\hline Min. Working Distance & $50 \mathrm{~mm}$ & $25 \mathrm{~mm}$ \\
\hline
\end{tabular}

GaAsP Photodiode was used as a sensor for measurement of radiant fluence. It has a spectral response in the range of $280-400 \mathrm{~nm}$ with the peak near $370 \mathrm{~nm}$. This type of sensor was chosen because it is sensible to wavelengths of light that are used for solidifying of common photopolymers.

Projector was fixed at the mobile platform, therefore distance between lens and projected area was able to change. Two different distances were chosen for measurement. These distances were set up to reach two different illuminated areas. Area of illuminated part of the platform has influence on precision of a printed model.

A sensor was installed on the console in front of projector on the projected area. Duration of all measurements was set to 200 seconds. Measurement started in reference point. After finishing of measurement in the first point sensor with a frame was moved to the second point. This routine was repeated for every chosen point on illuminated area.

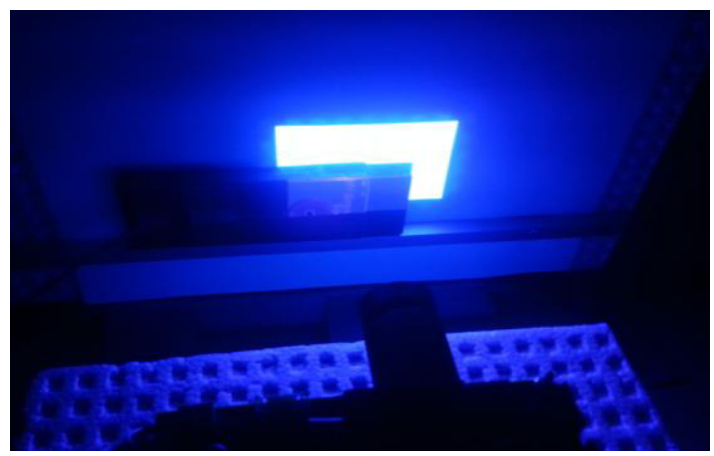

Figure 2. Image of measurement process of LightCrafter

Measurement for LightCrafter projector, which is shown on Figure 2, was provided for two areas with different distance between lens and projected area. It was made for getting information about influence of distance on radiant fluence. For Acer projector the one experiment with constant distance was enough, because during test radiant energy had relatively low level and providing of second experiment was useless, as soon as no additional information could be provided. 


\section{Results and discussion}

Data from a measurement are presented below. Figure 3 shows distribution of radiant energy for LightCrafter projector on printed area $80 \times 50 \mathrm{~mm}$. In this case one pixel (one mirror of DMD chip) has a size $62.5 \times 62.5 \mu \mathrm{m}$ on the projected surface. Precision of the printing on this area will be equal to a size of one pixel. Bottom side of Figure 3 shows 2D wire graph of radiant fluence. Maximal measured value for this case is equal to $156 \mathrm{~mJ} / \mathrm{cm}^{2}$ that corresponds to $100 \%$. Bottom part of figure shows, that the peak of radiant fluence is not in the center of printed area. Also it is important to note that the value of radiant fluence is significantly smaller in the corners of printed area. It signifies that process of solidification will not be even on the whole area. This effect is displayed better during printing with short time intervals of radiation. In this case some areas got not enough radiant energy and did not solidify properly. It can cause a damage of whole model, as it is shown on Figure 4. That's why effective printing space is smaller than illuminated area that is shown on Figure 3.

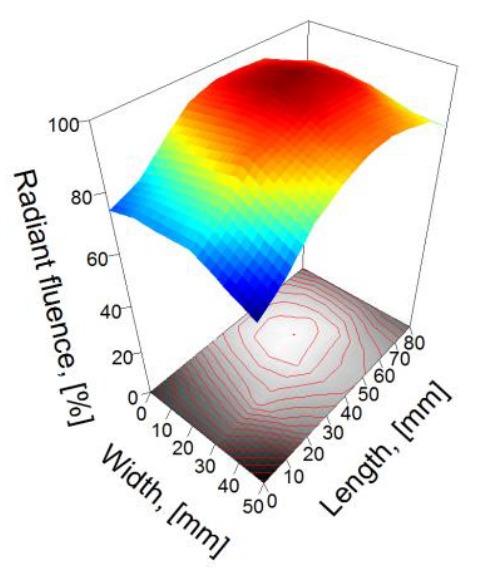

Figure 3. Relative radiant fluence of LightCrafter projector with precision of $62.5 \mu \mathrm{m}$

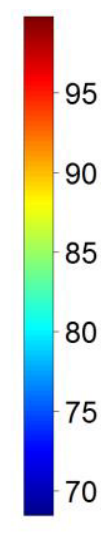

Figure 4. The damaged printed model

Increasing of effective printing space up to illuminated area is possible by rising a time of radiation for each layer. For this projector overheating of the platform was not noticed, because most of consumed energy were transformed to light beam in UV range.

Difficulties with overheating are common for not UV projectors. Visible spectrum, which is generated by Acer projector, has a low amount of UV light. It means, that big amount of energy, which is consumed by the projector, is used not for solidification of build material, but for heating of the platform, model and photopolymer solution. It can cause internal stress in built model, which may lead to mechanical defects, such as bending during or after printing. Also some parts of the model may remain non-solidified, then the resulting model becomes damaged, similarly to the model, shown on Figure 4.

Sometimes built models can unstick from the platform in the corners or even all over the entire surface. It can cause damaging of a tank with photopolymer. In this case using of manually added support structures can be effective. But in the case of DLP printers, it is not always useful and take more time for preprocessing.

In Figure 5 results of measurement of radiant fluence for the LightCrafter projector but on the different printing area are shown. In this case printing area is $40 \times 25 \mathrm{~mm}$ with precision of $31.25 \mu \mathrm{m}$. Distance between projector and sensor is lower, that's why amount of radiant energy is considerably higher, than in the first case. Consequently, unevenness is higher as well (in comparison to measurements on the larger area). Maximal radiant fluence in this case is $760 \mathrm{~mJ} / \mathrm{cm}^{2}$. Measurement was provided in the same number of points. 


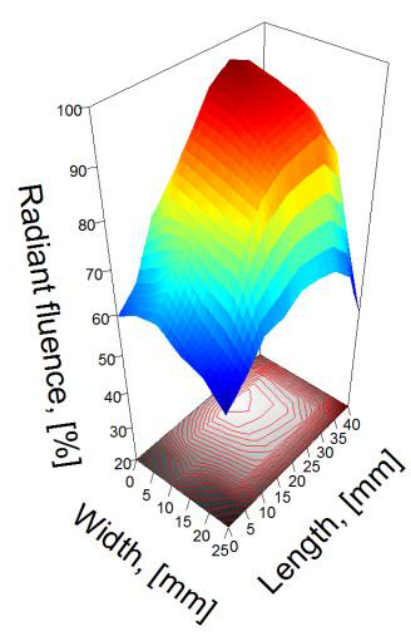

Figure 5. Relative radiant fluence of LightCrafter projector with precision of $31.25 \mu \mathrm{m}$

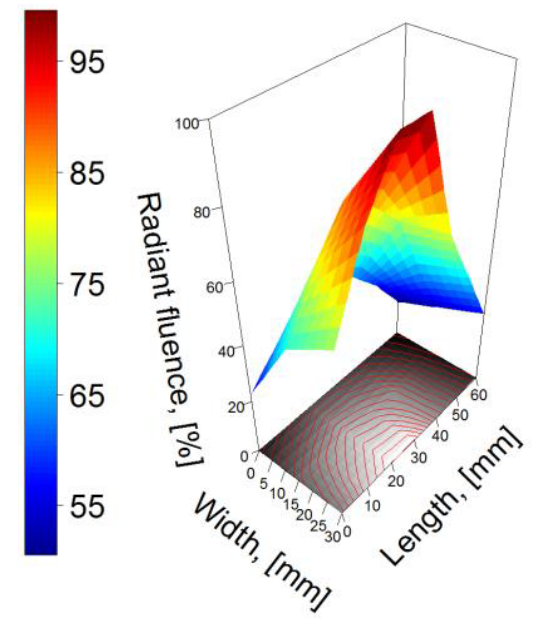

90

80

70

60

50

40

30

20

Figure 6 shows relative value of radiant fluence for the Acer projector. Printing area for this measurement is larger, than for the second case of LightCrafter projector (Figure 5). It is $59.4 \mathrm{~mm} \mathrm{x}$ $33.8 \mathrm{~mm}$. Precision remains the same as in previous case, $31.25 \mu \mathrm{m}$. It is caused by bigger DMD chip in Acer projector with resolution 1920 x 1080 mirrors. Maximum value of radiant fluence is 31 $\mathrm{mJ} / \mathrm{cm}^{2}$. The bottom part of Figure 6 shows, that the peek radiant energy is directly at the verge of printing area. It is caused by the offset of the Acer projector. It causes nonuniform solidifying effect at some distance from center and decreases effective printing area in comparison with a LightCrafter projector.

\section{Conclusions}

Common widespread Acer DLP projector generates just 4\% of radiant energy in comparison with industrial LightCrafter projector. Measurements showed that the peak of radiant energy for the LightCrafter projector is closer to the center of printing area as compared to the Acer projector. This option can rise effective printing area, even in comparison with Acer projector which has higher resolution of DMD chip and larger printing area. And the last, but not the least important parameter of tested projectors is energy consumption, which is for Acer projector higher, as compared to the LightCrafter projector.

All these advantages of industrial projector not only make 3D printer more effective, but also improve quality of printed models. Reliability of $3 \mathrm{D}$ printer can be increased, since an undesired heating of the platform can be weakened or even illuminated.

\section{Acknowledgement}

The research reported in this paper was supported by targeted support for specific university research within the student grant competition TUL (Project 21071 - Development and prototype production of compact DLP 3D printer). 


\section{References}

1. J.M. Waller, B.H. Parker, K.L. Hodges, E.R. Burke, J.L. Walker, E.R. Generazio, Nondestructive Evaluation of Additive Manufacturing (Langley Research Center, Hampton, 2014)

2. T. WohlDer, T. Gornet, Wohlers Report 2016:3D Printing and Additive Manufacturing State of the Industry, (Wohlers Associates Inc., USA, 2016)

3. C.W. Hull, Apparatus for production of three-dimensional objects by stereolithography, U.S Patent 4575330 (1986)

4. $\quad$ L.J. Hornbeck, Frame addressed spatial light modulator, U.S. Patent 4615595 (1986)

5. A. de Blas Romero, M. Pfaffinger, J. Mitteramskogler et al. Int. J. Adv. Manuf. Technol. (2016) doi:10.1007/s00170-016-8856-1

6. M.L. Griffith, J.W. Halloran, J. Am. Ceram. Soc., 79, 10 (1996)

7. R. Gmeiner, G. Mitteramskogler, J. Stampfl, Int. J. Appl. Ceram. Technol., 12, 1 (2015) DOI:10.1111/ijac. 12325

8. R. Felzmann, S. Gruber, G. Mitteramskogler, P. Tesavibul, A.R. Boccaccini, R. Liska, J. Stampfl, Adw. Eng. Mat. 14, 12 (2012) DOI: 10.1002/adem.201200010

9. G. Mitteramskogler, R. Gmeiner, R. Felzmann, S. Gruber, C. Hofstetter, J. Stampfl, J. Ebert, W. Wachter, J. Laubersheimer, Add. Manuf., 1-4, (2014)

10. R. Holtrup, Design and construction of a multi-material 3D DLP printer, (University of Twente, Netherlands, 2015)

11. V. Bernabé-Zafón, M. Beneito-Cambra, E.F. Simó-Alfonso, J.M. Herrero-Martínez, Journal of Chromatography A, 1217 (2010) 